\title{
L-FVM for Unsteady Seepage Flow in Low Permeability Coalbed
}

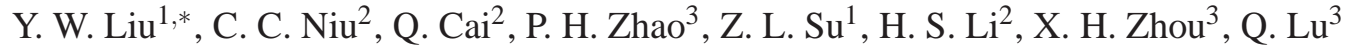 \\ ${ }^{1}$ Key Laboratory of Environment Mechanics, Institute of Mechanics, Chinese Academy of Sciences, Beijing 100190, \\ China \\ ${ }^{2}$ Beijing Technology and Business University, Beijing 100048, China \\ ${ }^{3}$ Coalbed Methane Ltd. Company, Petrochina, Beijing 100028, China
}

Email: Lywu@imech.ac.cn

\begin{abstract}
The significant feature of coalbed in China is the low permeability. A new unsteady seepage flow model is developed for the low permeability coalbed by considering the startup pressure gradient and methane desorption effect. Since the complexity of the problem, a new method which we call it "L-FVM" is developed, based on comparing the normal numerical calculation methods and comprehension research on FVM. The results show that L-FVM has the same precission but higher calculating velocity than normal FVM. This result is very important for monitoring the area pressure drawdown in coalbed methane engineering.
\end{abstract}

Key words: Coalbed, low permeability, adsorption, FVM, seepage flow

\section{INTRODUCTION}

The resource of coalbed methane is $36.8 \times 10^{12} \mathrm{~m}^{3}$ in China, and it takes the third place in the world [1]. But the significant feature of coalbed in China is the low permeability distinguished with the coalbed in the USA. Some technology used well abroad is not suitable in china. In low permeability coalbed, fluid flow must overcome the startup pressure gradient. A new unsteady seepage flow model is developed for the low permeability coalbed by considering the startup pressure gradient and methane desorption effect. Since the existents of the startup pressure gradient term and the methane desorption effect term, it is too difficult to solve the problem by using analytical methods.

FVM is a numerical method which developed from the late 1950s after Godunov [2]. In 1980s [3], the research and application of FVM take a long way since the development of mesh technique, especially the unstructured mesh. During this period [3], total variation diminishing (TVD) scheme and a serials effective methods were generated such as Upwind-FVMGudunov-FVMRiemann-FVM and TVD-FVM etc, but they all worked on the calculation structure. Here, based on comparing results of the normal numerical calculation methods and comprehension research on FVM, a new FVM named L-FVM is developed by the Laplace space transformation.

\section{MATHEMATICAL MODEL}

By considering the low permeability feature and the desorption phenomena of the coalbed methane, a new mathematical model is developed for the unsteady fluid flow in the low permeability coalbed.

Governing equation of fluid flow in the coalbed

$$
\frac{\partial^{2} p_{\mathrm{D}}}{\partial x_{\mathrm{D}}^{2}}+\frac{\partial^{2} p_{\mathrm{D}}}{\partial y_{\mathrm{D}}^{2}}+\alpha_{1 \mathrm{D}}+\alpha_{2 \mathrm{D}}\left(p_{\mathrm{D}}-p_{\mathrm{CD}}\right)+\frac{\lambda_{\mathrm{PD}}}{r_{\mathrm{D}}}=\frac{1}{C_{\mathrm{D}} e^{2 S}} \frac{\partial p_{\mathrm{D}}}{\partial T_{\mathrm{D}}}, \quad x_{\mathrm{D}}, y_{\mathrm{D}} \in \Omega
$$

Innerboundaryconditions : $\left.\quad\left(\frac{\partial p_{\mathrm{D}}}{\partial x_{\mathrm{D}}} \cos \theta+\frac{\partial p_{\mathrm{D}}}{\partial y_{\mathrm{D}}} \sin \theta\right)\right|_{r_{\mathrm{D}}=1}=-1-\lambda_{\mathrm{PD}}+\frac{\mathrm{d} p_{\mathrm{D} w}}{\mathrm{~d} T_{\mathrm{D}}}$ 
Initialconditions : $\left.\quad p_{\mathrm{D}}\right|_{T_{\mathrm{D}}}=0$

Outer boundary conditions:

Forinfiniteboundary : $\left.\quad p_{\mathrm{D}}\right|_{x_{\mathrm{D}}, y_{\mathrm{D}} \rightarrow \infty}=0$

Forconstantpressureboundary : $\left.p_{\mathrm{D}}\right|_{\Gamma_{\mathrm{out}}}=0$

Forclosedboundary : $\left.\frac{\partial p_{\mathrm{D}}}{\partial n}\right|_{\Gamma_{\text {out }}}=0$

Where: $p_{\mathrm{D}}$ is the dimensionless pressure, $T_{\mathrm{D}}$ is the dimensionless time, $x_{\mathrm{D}}, y_{\mathrm{D}}$ is the dimensionless position in the coalbed, $p_{\mathrm{CD}}$ is the dimensionless critical adsorption pressure, $\alpha_{1 \mathrm{D}}$ is the dimensionless steady adsorption coefficient, $\alpha_{2 \mathrm{D}}$ is the dimensionless unsteady adsorption coefficient, $\lambda_{\mathrm{PD}}$ is the dimensionless start-up pressure gradient, $r_{\mathrm{D}}$ is the dimensionless distance form the center of the wellbore, $\theta$ is the angle value, $\Gamma_{\text {out }}$ indicate the outer boundary, $\Omega$ indicate the research region, $C_{\mathrm{D}} e^{2 S}$ is the dimensionless combined coefficient.

\section{L-FVM METHOD}

At first, we should transform the mathematical model into Laplace space. The governing equation of fluid flow in the coalbed is

$\frac{\partial^{2} \bar{p}_{\mathrm{D}}}{\partial x_{\mathrm{D}}^{2}}+\frac{\partial^{2} \bar{p}_{\mathrm{D}}}{\partial y_{\mathrm{D}}^{2}}+\frac{\alpha_{1 \mathrm{D}}}{z}+\alpha_{2 \mathrm{D}}\left(\bar{p}_{\mathrm{D}}-\frac{\overline{P_{\mathrm{CD}}}}{z}\right)+\frac{\lambda_{\mathrm{PD}}}{z r_{\mathrm{D}}}=\frac{z}{C_{\mathrm{D}} e^{2 S}} \bar{p}_{\mathrm{D}}$

The equations of L-FVM can be derivted as following

$$
\begin{gathered}
\sum_{\text {all perface }}\left[\frac{\Delta A_{i}}{n_{i} \cdot e_{\xi_{i}} \cdot \Delta \xi_{i}}\left(\bar{P}_{A}^{n+1}-\bar{P}_{P}^{n+1}\right)-\frac{e_{\xi_{i}} \cdot e_{\eta_{i}} \cdot \Delta A_{i}}{n_{i} \cdot e_{\xi_{i}} \cdot \Delta \eta_{i}}\left(\bar{P}_{b}^{n+1}-\bar{P}_{a}^{n+1}\right)\right]+ \\
{\left[\frac{\alpha_{1 \mathrm{D}}}{z}+\alpha_{2 \mathrm{D}}\left(\bar{p}_{P}^{n+1}-\frac{\bar{P}_{c D}}{z}\right)+\frac{\lambda_{\mathrm{PD}}}{z r_{\mathrm{D}}}\right] \Delta V=\frac{z}{C_{\mathrm{D}} e^{2 S}} \bar{p}_{P}^{n+1} \Delta V}
\end{gathered}
$$

\section{RESULTS AND ANALYSIS}

1. Well test type curve The log-log type curve for the mathematical model is showed in Figure 1. In Figure 1, it shows that the type curve can be devided into 6 stages. (1) In the wellbore control stage, pressure and pressure derivative curves are straight lines with slope equals to 1. (2) A transient flow stage. (3) The skin effect stage, there may apear a variable "bulge" in the pressure derivative curve with different skin values. (4)The radial flow period, the pressure derivative curve maybe a horizontal line with the value equals to 0.5. (5) The startup pressure gradient effect stage, pressure and pressure derivative curves both holding up with different startup pressure gradient values. (6) CBM desorption and outer boundary effect stage, for the circle closed boundary, the pressure and pressure derivative curves are straight lines with slope equals to 1 .

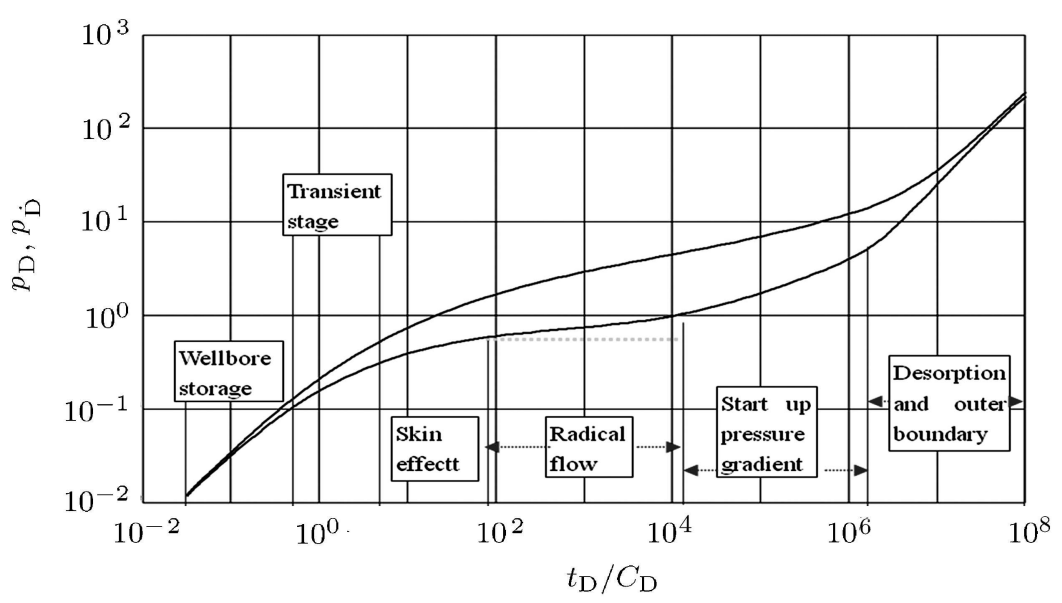

Figure 1: The type curve for the mathematical model 
2. Effect of startup pressure gradient In the condition of closed boundary, let the combined coefficient $C_{\mathrm{D}} e^{2 S}=10$, desorption coefficient $\alpha_{1 \mathrm{D}}=0, \alpha_{2 \mathrm{D}}=0$, critical desorption pressure $P_{\mathrm{CD}}=0$ respectively, and the startup pressure gradient $\lambda_{\mathrm{PD}}=0,0.01,0.1$ respectively, the effect of startup pressure gradient $\lambda_{\mathrm{PD}}$ on well test type curves can be seen clearly in Figure 2. In Figure 2, it shows that the more larger the startup pressure gradient, the more higher the type curves holding up.

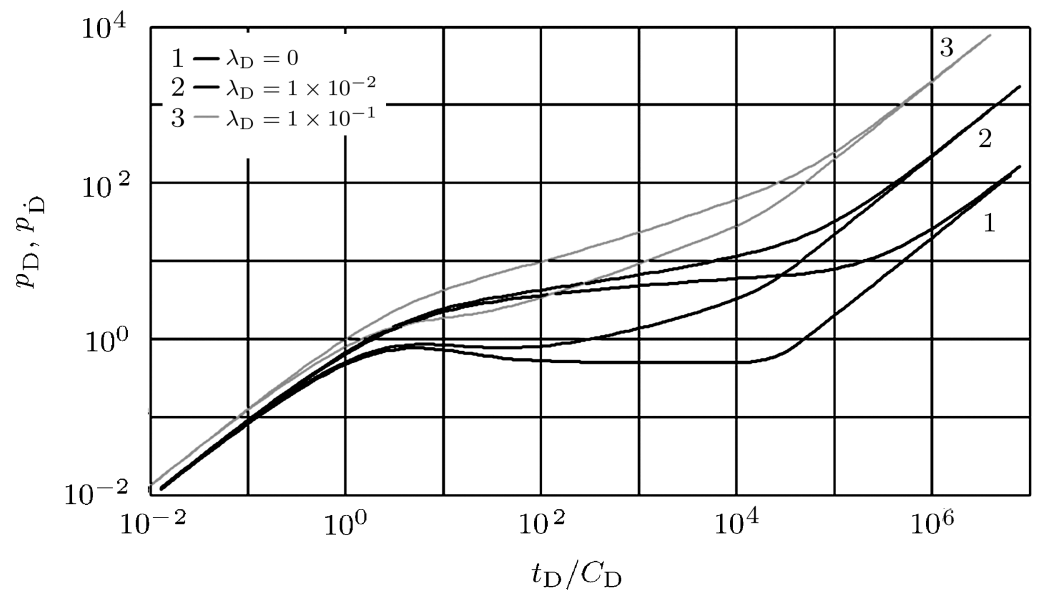

Figure 2: Type curves with different startup pressure gradients

\section{COMPARISON}

1. Comparison of the pressure profile in coalbbed In order to verify the L-FVM method, we compared the calculation results of the pressure profile in coalbed with that of the normal FVM method. The results show in Figure 3.

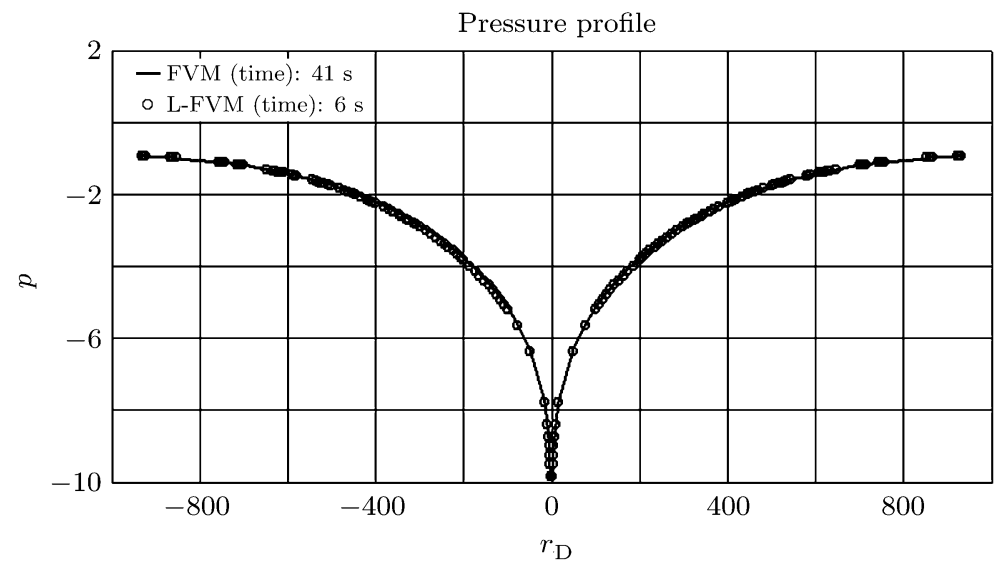

Figure 3: Pressure profiles between FVM and L-FVM

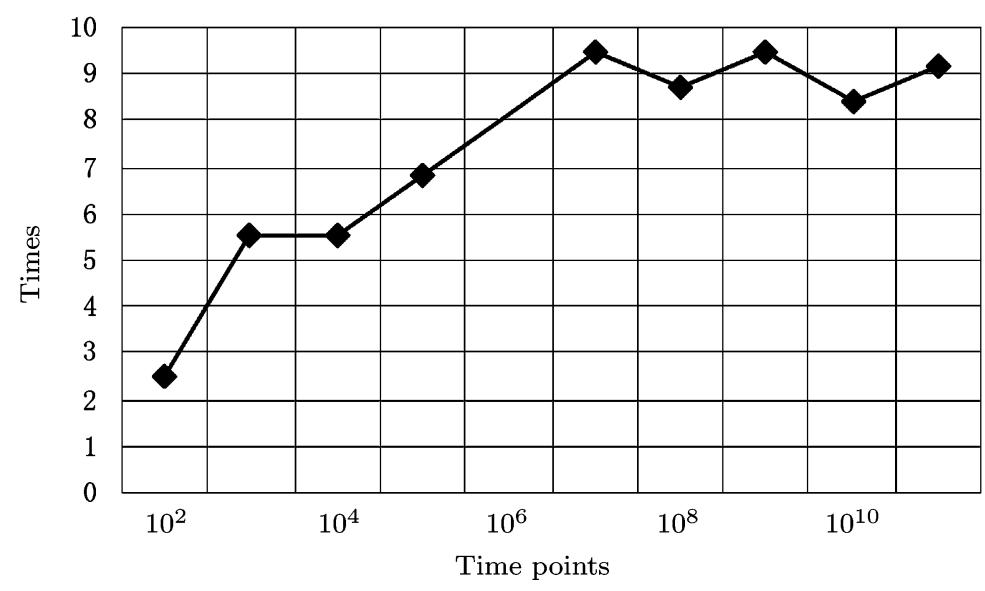

Figure 4: Comparison results of calculating velocity between FVM and L-FVM 
2. Comparison of the calculating time To evaluate the area pressure drawdown effects, the seepage field must be calculated. L-FVM takes the advantage of high calculating velocity. The comparison results of computing velocity between FVM and L-FVM on HP 8700 computer are showed in Figure 4.

\section{CONCLUSSION}

(1) A new mathematical model is developed for unsteady fluid flow in the low permeability coalbed, by considering the low permeability feature and the desorption phenomena of the coalbed methane. The effect of start-up pressure gradient on well test type curve is discussed in detail.

(2) A new calculation method L-FVM is founded by translating the equations into Laplace space and solved on unstructured triangular mesh. The validity and precision of L-FVM are verified by comparing the results with the normal FVM.

(3) By comparing the computing velocity with the normal FVM on HP 8700 computer, after $T_{\mathrm{D}}>10^{3}$ the results show that L-FVM is 5 times faster than FVM.

\section{Acknowledgements}

The support of National S\&T Major Project of China (2009ZX05038001) is gratefully acknowledged.

\section{REFERENCES}

1. Chen HN, Zhang WD, Xie LY, et al. Reserves and development status of global unconventional natural gas. FaultBlock Oil \& Gas Field, 2010; 17(4): 439-442

2. Godunov SK. A finite difference method for numerical computation and discontinuous solutions of the equations of fluid dynamics. Mat S B, 1959; 47: 271

3. Versteeg h K, Malalaselera W. An Intoduction to Computational Fluid Dynamics, The Finite Volum Method. Pearson Prentice Hall, Second Edition, 2007 